\title{
Sugar intake and dental decay: results from a national survey of children in Scotland
}

\author{
Lindsey F. Masson ${ }^{1}$, Alison Blackburn ${ }^{1}$, Christine Sheehy ${ }^{2}$, Leone C. A. Craig ${ }^{1,3}$, Jennie I. Macdiarmid ${ }^{3}$, \\ Bridget A. Holmes ${ }^{4}$ and Geraldine McNeill ${ }^{1,3}$ \\ ${ }^{1}$ Institute of Applied Health Sciences, University of Aberdeen, Polwarth Building, Foresterhill, Aberdeen AB25 $2 Z D$, UK \\ ${ }^{2}$ Scottish Centre for Social Research, Edinburgh, UK \\ ${ }^{3}$ Rowett Institute of Nutrition and Health, University of Aberdeen, Aberdeen, UK \\ ${ }^{4}$ Nutritional Sciences Division, King's College London, London, UK
}

(Received 17 November 2009 - Revised 24 May 2010 - Accepted 25 May 2010 - First published online 19 July 2010)

\begin{abstract}
The aim of this analysis was to investigate the strength of the association between sugar intake and treatment for dental decay in children in Scotland, and the impact of tooth brushing frequency on this association. The Survey of Sugar Intake among Children in Scotland was carried out in 2006 in those aged 3-17 years. Diet was assessed using the Scottish Collaborative Group FFQ, and interviews were carried out by trained fieldworkers who asked about dental health. A total of 1700 interviews were carried out, and $1512 \mathrm{FFQ}$ were returned. Of the children, $56 \%$ had received treatment for decay (fillings or teeth removed due to decay). Intake of non-milk extrinsic sugars (NMES), but not total sugar, increased the risk of having had treatment for decay: adjusted OR 1.84 (95\% CI 1.28, 2.64) for the highest ( $\geq 20 \cdot 0 \%$ food energy) $v$. lowest $(\leq 14.8 \%$ food energy) tertile of NMES intake. This raised risk remained in children who reported brushing their teeth at least twice a day. Compared with children who reported brushing their teeth at least twice a day and were in the lowest tertile of NMES intake, children who reported brushing their teeth once a day or less and were in the highest tertile of NMES intake were over three times more likely to have received treatment for decay (adjusted OR 3.39, $95 \%$ CI 1.97, 5.82). In order to improve dental health in children in Scotland, dental health strategies must continue to stress the importance of both reduced NMES intake and good oral hygiene.
\end{abstract}

Sugar: Dental decay: Children: Scotland

Dental caries (decay) occurs when bacteria living in dental plaque ferment dietary carbohydrate, producing acid that lowers plaque $\mathrm{pH}$. When the $\mathrm{pH}$ at the tooth surface falls, demineralisation occurs, with calcium and phosphates diffusing out of the tooth enamel. Repeated exposure to reduced plaque $\mathrm{pH}$ results in sub-surface softening of the enamel, and the lesion can progress to form a carious cavity ${ }^{(1)}$.

Development of caries is influenced by previous disease, use of fluoride, plaque control, salivary flow, medical history and diet, which in turn are influenced by social factors. The dietary factors include the amount of sugar consumed, sugar concentration of food, physical form of carbohydrate, oral retentiveness (length of time teeth are exposed to reduced plaque $\mathrm{pH}$ ), frequency of eating meals and snacks, length of interval between eating and sequence of food consumption ${ }^{(1)}$. In particular, the amount and frequency of consumption of non-milk extrinsic sugars (NMES) are considered to be the major causes of dental caries in the $\mathrm{UK}^{(2)}$. NMES are those sugars which are not located within the cellular structure of a food, and exclude the sugar in milk (almost all lactose) which is not thought to contribute to dental caries ${ }^{(2)}$. Therefore, NMES includes all sugars in fruit juices as well as table sugar, honey and the sucrose, glucose and glucose syrups added to foods.

Good oral hygiene is central to the strategy for improving children's dental health. It is recommended that teeth require frequent brushing (at least twice a day) from an early age ${ }^{(1,3)}$, and it has been suggested that frequent brushing (with a fluoride toothpaste) may have greater impact on the prevention of caries in young children than restricting sugary foods ${ }^{(4)}$. Socio-economic inequalities in tooth brushing habits persist, both in Scotland ${ }^{(5)}$ and in many other countries ${ }^{(6)}$, with family affluence associated with a higher prevalence of frequent tooth brushing.

Children in Scotland have poorer dental health than children in England or Wales ${ }^{(7,8)}$. Within Scotland, dental health has changed little between 1998 and $2003^{(9)}$, although the proportion of boys and girls brushing teeth at least twice a day has risen between 1998 and 2006 (from 56-57\% to $63-66 \%$ in 11-, 13- and 15-year-old boys, and from $67-78 \%$ to $78-82 \%$ in $11-, 13-$ and 15 -year-old girls $)^{(5)}$ Poor dental health in children may lead to physical pain, embarrassment and absences from school as well as to demands on health care budgets ${ }^{(3)}$. Over $£ 200$ million of

Abbreviation: NMES, non-milk extrinsic sugars.

* Corresponding author: Dr Lindsey F. Masson, fax +44 1224 550925, email lindsey.masson@abdn.ac.uk 
public money is spent on Scotland's dental services each year, and the majority of this is spent on treating dental caries ${ }^{(3)}$. In the past year within Scotland, the total money spent on children's dental care increased from $£ 57$ to $£ 63$ million ${ }^{(10)}$.

In 2006, the Food Standards Agency, Scotland, commissioned a survey to investigate the diet of children across Scotland with the primary aim of comparing NMES intake with the Scottish dietary target of $<10 \%$ of total energy ${ }^{(11)}$. This target was based on the UK dietary reference value of a population average intake of $\leq 10 \%$ of total energy $(\leq 11 \% \text { of food energy })^{(12)}$. The survey found that mean NMES intake was much higher than recommendations at $17.4 \%$ of food energy in children aged $3-17$ years ${ }^{(13)}$. Intakes were similar in boys and girls, but increased significantly with age, from $15.8 \%$ of food energy in $3-7$ year olds up to $19.1 \%$ of food energy in 12-17 year olds ${ }^{(13)}$.

The aim of the present analysis was to assess the strength of the association between intakes of sugars and foods that are significant sources of NMES and treatment for dental caries with adjustment for confounding factors, and the impact that frequent tooth brushing has on this association, in children aged 3-17 years living in Scotland. The data used were from the recent national Survey of Sugar Intake among Children in Scotland ${ }^{(13)}$.

\section{Methods}

Full details of the methodology for the Survey of Sugar Intake among Children in Scotland can be found in the final report (the supplementary material for this article can be found at http://www.journals.cambridge.org/bjn $)^{(13)}$. A brief summary is provided here.

\section{Sample}

A named sample of 2800 Scottish children aged 3-16 years on 1 May 2006 was drawn from eighty postcode sectors across Scotland using the Department of work and pensions child benefit records. Three hundred and two children were excluded by the Department of Work and Pensions as sensitive cases or because they were involved in other surveys, and 146 parents/guardians chose to opt out after an initial invitation letter had been sent. An FFQ, used to estimate habitual dietary intake, was sent to the remaining 2352 children.

Fieldworkers arranged a home visit during which they checked the completed FFQ. If the FFQ had not been completed, a further copy was left with the family with a reply-paid envelope. Height and weight were measured by the fieldworkers, and data on demographic characteristics, physical activity and dental health were collected by computer-assisted personal interview using questions that were the same as those used in the 2003 Scottish health survey ${ }^{(9)}$. The dental health module included questions on attendance at a dentist, treatment received from a dentist and frequency of toothbrushing. For children aged under 12 years, the interview was conducted with the parent or guardian. Children aged 12 years and over were asked to complete the questions related to dental health themselves with help from a parent or guardian as necessary.

Fieldwork was carried out between May and September 2006. A total of 1700 interviews were conducted and
1512 FFQ were returned. Both interview and FFQ data were provided for 1491 children (66\% of those invited to participate).

The present study did not require the approval of a National Health Service research ethics committee, but it was carried out in accordance with the research governance and quality assurance policies of the Scottish Centre for Social Research and the Institute of Applied Health Sciences at the University of Aberdeen (http://www.abdn.ac.uk/iahs/research-governance/governance.shtml). After the initial opt out, consent was given by completion of the FFQ and written consent was obtained for the interview and measurements.

\section{Dietary data}

The Scottish Collaborative Group $\mathrm{FFQ}^{(14)}$ was used to estimate the habitual intake of foods and nutrients. Two new versions were developed for this survey: version $\mathrm{C} 2$ for children aged 3-11 years designed for completion by a parent/guardian with help from the child, and version C3 for young people aged 12-17 years for completion by the young person with help from their parent/guardian. Version C2 lists 140 foods or drinks with a measure defined for each item, and version C3 includes six additional items covering intake of coffee and alcoholic drinks. Participants were asked to estimate the frequency and amount of each food or drink consumed by selecting one of nine options, ranging from 'rarely or never' to 'seven or more measures per day'. The relative validity of the FFQ was assessed in a subgroup of the study population who also completed a $4 \mathrm{~d}$ non-weighed diet diary ${ }^{(13)}$.

Data were entered manually, and a $10 \%$ check of the data entry found an error rate of $0.4 \%$. FFQ items were divided into thirty-three food or drink groups, modified slightly from the Low-Income Diet and Nutrition Survey ${ }^{(15)}$; in a few instances, the grouping of foods on the FFQ led to minor differences in the definitions of these groups. Energy and nutrient intakes were estimated from an in-house calculation package based on the Food Standards Agency National Diet and Nutrition Survey nutrient database, which uses the following criteria to estimate NMES content of foods ${ }^{(16)}$ : all sugars in fresh fruit and vegetables are classified as intrinsic, and lactose, whether present in a milk product or not, is considered a milk sugar. All sugars in fruit juices as well as table sugar, honey and the sucrose, glucose and glucose syrups added to foods are classified as extrinsic. Sugars naturally present in foods that are canned, stewed, dried or used in preserves are classified to be half extrinsic and half intrinsic. Added sugars or syrup in preserves are classified as extrinsic sugars.

\section{Socio-economic status and overweight}

Socio-economic status was assessed using the 2006 Scottish index of multiple deprivation, which is a measure of neighbourhood socio-economic status based on twenty-seven indicators from seven domains (income, housing, employment, health, crime, education and access to services) ${ }^{(17)}$. Measured weight and height were used to calculate BMI $\left(\mathrm{kg} / \mathrm{m}^{2}\right)$, which was compared with the UK 1990 centile charts $^{(18)}$. Overweight (but not obese) was defined as BMI $\geq 85$ th centile but $<95$ th centile, and obesity was defined as $\mathrm{BMI} \geq 95$ th centile. 


\section{Data analysis}

Statistical analysis was carried out using Stata/SE 9.2 (Stata Corporation, Lakeway Drive, College Station, TX, USA). The data were weighted so that the estimates generated from the responding sample reflected more accurately the characteristics of the population of children aged 3-16 years on 1 May 2006 in Scotland. Weightings were calculated to take account of selection and non-response bias, and then these composite weights were adjusted to create a calibration weight ${ }^{(13)}$.

Differences between children who had never been treated for decay and those who had received treatment for decay were assessed using the Pearson $\chi^{2}$ statistic for categorical variables, which was corrected for the survey design ${ }^{(19)}$, or using a $t$-test for continuous variables.

After excluding fifty-one (3\%) FFQ with more than ten missing responses and seventy (5\%) FFQ with extreme energy intakes (below the 2.5 percentile or above the 97.5 percentile of energy intake for the age groups 3-7, 8-11 and 1217 years), dietary data were available for 1391 children. Both dietary data and data on treatment for decay were available for 1329 children. Sugar and food intakes expressed as g/d were adjusted for total energy using the nutrient residual $\operatorname{method}^{(20)}$, and sugar intakes were also expressed as a percentage of food energy assuming the energy content of $16 \mathrm{~kJ} / \mathrm{g}$. The food groups that were analysed for their association with treatment for decay were those that contributed at least $10 \%$ to the intake of total sugars or NMES, and also crisps and savoury snacks. This analysis is based on both consumers and non-consumers of these foods. Sugar and food intakes were categorised into tertiles of intake: T1 (lowest), T2 (medium) and T3 (highest). Sugar and food intakes are reported to one decimal place, although a second decimal place was used to define cut-offs for tertiles.

Logistic regression was used to compute crude and adjusted OR $(95 \% \mathrm{CI})$ for each tertile of intake, using the lowest tertile of intake as the reference group. A model for the risk of treatment for decay was developed using stepwise regression to evaluate the effects of including the variables which were significantly different between children who had never been treated for decay and those who had received treatment for decay: age (at interview), ethnicity, BMI classification, Scottish index of multiple deprivation quintile, type of teeth (milk or second teeth), age of first attendance at the dentist, reason for the first visit to the dentist, treatment to stop decay (painting and/or sealing), treatment from an orthodontist, age when first brushed teeth first, frequency of tooth brushing, type of toothpaste, ever visited a dental surgery, whether had false teeth and use of fluoride supplements. Variables with $P>0.1$ from the Wald test for significance were removed from the model.

\section{Results}

\section{Subject characteristics}

The children were aged between 3 and 17 years at interview. The majority $(97 \%)$ of children were white, and $51 \%$ were male. Of the children, $14 \%$ were classified as overweight (but not obese), and $17 \%$ were obese. Most of the children (97\%) had attended the dentist at least once. At age 3-7 years, $94 \%$ of the children had attended the dentist, and this proportion rose to $99 \%$ by age $12-17$ years.

\section{Treatment for decay}

Over half $(56 \%)$ of all children had reported receiving treatment for decay. Almost a third $(31 \%)$ had had fillings but no teeth extracted due to decay, $7 \%$ had had teeth extracted due to decay but no fillings, and another $17 \%$ had had both fillings and teeth extracted due to decay.

Children who reported that they had been treated for decay were more likely to be older $(P<0.001)$, overweight or obese $(P=0.031)$, from more deprived areas $(P<0.001)$, and to have their second (or adult) teeth $(P<0.001)$ compared with children who reported that they had never received treatment for decay (Table 1). In addition, those who had been treated for decay were more likely to have been older when they first attended the dentist $(P<0 \cdot 001)$, to have attended the dentist for the first time because of trouble with their teeth $(P<0 \cdot 001)$, and to have received other dental treatment such as painting and/or sealing $(P<0 \cdot 001)$ and orthodontics $(P=0 \cdot 015)$. Tooth brushing habits were also associated with treatment for decay: those who reported that they had been treated for decay were older when they first brushed their teeth $(P<0 \cdot 001)$, and less likely to brush their teeth at least twice a day $(P<0 \cdot 001)$. Most of the children $(97 \%)$ reported using a fluoride toothpaste, while only $0.3 \%$ of all children reported taking fluoride supplements. All children who reported taking fluoride supplements also reported using a fluoride toothpaste.

\section{Frequency of tooth brushing}

Tooth brushing frequency differed significantly by age, sex and Scottish index of multiple deprivation quintile $(P<0.001$ for all). Younger children were more likely to report brushing their teeth frequently (at least twice a day) than older children $(76 \%$ in $3-7$ year olds, $68 \%$ in $8-11$ year olds, and $63 \%$ in 12-17 year olds), and girls were more likely to report brushing their teeth frequently than boys (74 v. 63\%). Children in the least deprived Scottish index of multiple deprivation quintile were also more likely to report brushing their teeth frequently $(80 \%)$ compared with children in the most deprived quintile $(55 \%)$. Frequency of tooth brushing also differed by NMES intake: $76 \%$ of children in the lowest tertile of intake ( $\leq 14.8 \%$ food energy) reported brushing their teeth frequently compared with $65 \%$ of children in the highest tertile of intake $(\geq 20.0 \%$ food energy) $(P=0 \cdot 003)$.

\section{Intakes of sugar and significant food sources of non-milk extrinsic sugars and risk of treatment for decay}

Table 2 shows the crude and adjusted OR for the risk of having had treatment for decay (based on self-report) associated with sugar intake. Total sugar intake, as either $\mathrm{g} / \mathrm{d}$ or $\%$ food energy, was not significantly associated with having had treatment for decay. In contrast, NMES intake significantly increased the risk of having had treatment for decay, although OR were much attenuated following adjustment for potential confounding factors: adjusted OR $1.84(95 \% \mathrm{CI}$ $1.28,2.64$ ) for the highest ( $\geq 20.0 \%$ food energy) $v$. lowest ( $\leq 14.8 \%$ food energy) intake. Sucrose intake also significantly increased the risk of having had treatment for decay 
Table 1. Subject characteristics, dental history and oral hygiene habits, by treatment for decay (Mean values with their standard errors or percentages)

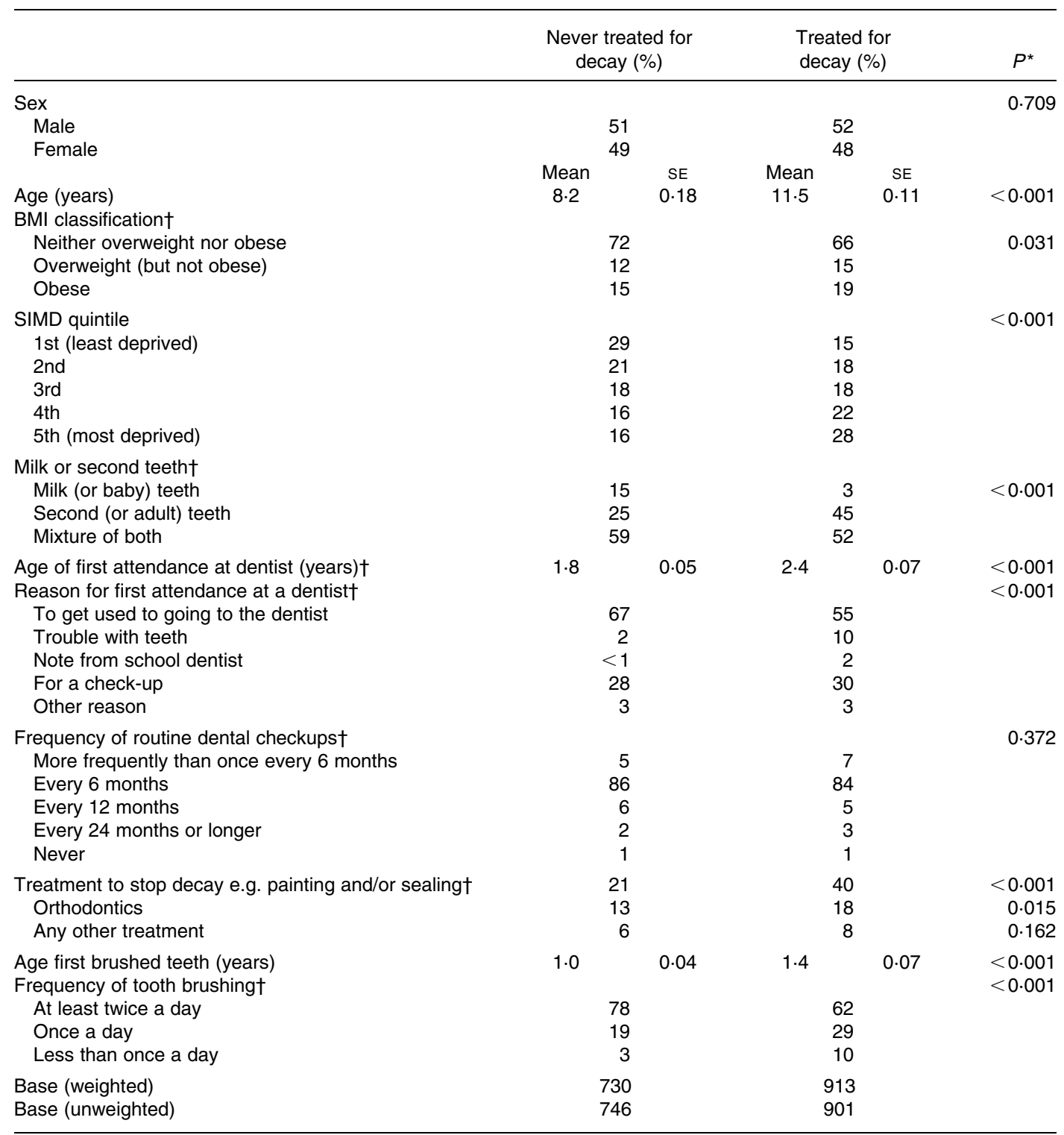

SIMD, Scottish index of multiple deprivation

${ }^{*} P$ for difference.

† Subjects with missing data excluded.

(adjusted OR 1.54, 95\% CI 1.08, 2.19) for the highest $v$. lowest intake (as percentage of food energy). In contrast, the highest intakes of intrinsic and milk sugars, fructose and lactose were significantly associated with a reduced risk of having had treatment for decay. Glucose and maltose intakes were not significantly associated with risk following adjustment (Table 2).

The main contributors to NMES intake in this population were non-diet soft drinks (23\%); confectionery (15\%); biscuits, cakes and pastries (13\%); yogurt and fromage frais $(10 \%)$; and fruit juice (including smoothies) $(10 \%)$. The main sources of total sugars were whole fruit $(16 \%)$; non-diet soft drinks (15\%); confectionery $(10 \%)$; and milk and cream (10\%). Of these food groups, only non-diet soft drinks and confectionery were positively associated with the risk of having had treatment for decay (Table 3). Children in the highest tertile of confectionery intake had double the risk compared with those in the lowest tertile of intake (adjusted OR 1.97, 95\% CI 1.39, 2.78). In addition, children with the medium or highest intake of crisps and savoury snacks were at significantly raised risk of having had treatment for decay compared with individuals with the lowest intake $(P=0.006$ for trend).

Fruit juice intake was significantly inversely associated with the risk of having had treatment for decay: adjusted OR 0.55 (95\% CI 0.37, 0.80) for the highest $v$. the lowest tertile of intake (Table 3). Similarly, children with both the medium and highest intakes of milk and cream were at significantly reduced risk of having received treatment for decay compared with children in the lowest tertile of intake. 
Table 2. Sugar intake and risk of having had treatment for decay ( $n$ 1329)

(Odds ratios and $95 \%$ confidence intervals)

\begin{tabular}{|c|c|c|c|c|c|c|c|c|c|c|}
\hline \multirow[b]{2}{*}{ Tertile } & \multicolumn{5}{|c|}{$\mathrm{g} / \mathrm{d}$} & \multicolumn{5}{|c|}{$\%$ Food energy } \\
\hline & Intake ${ }^{*} \dagger$ & Crude OR & $95 \% \mathrm{Cl}$ & Adjusted OR $\ddagger$ & $95 \% \mathrm{Cl}$ & Intake† & Crude OR & $95 \% \mathrm{Cl}$ & Adjusted OR $\ddagger$ & $95 \% \mathrm{Cl}$ \\
\hline \multicolumn{11}{|c|}{ Total sugars } \\
\hline T1 & $\leq 138.9$ & 1.00 & & 1.00 & & $\leq 26.6$ & 1.00 & & 1.00 & \\
\hline T2 & $138.9-161.5$ & 1.15 & $0.86,1.53$ & 1.25 & $0.87,1.80$ & $26 \cdot 6-31 \cdot 7$ & 1.01 & $0.76,1.33$ & $1 \cdot 15$ & $0.78,1.70$ \\
\hline T3 & $\geq 161.5$ & 1.14 & $0.86,1.51$ & 1.05 & $0.74,1.49$ & $\geq 31.7$ & $1 \cdot 21$ & $0.91,1.61$ & $1 \cdot 10$ & $0.77,1.57$ \\
\hline$P$ (trend) & & 0.345 & & 0.759 & & & 0.194 & & 0.576 & \\
\hline \multicolumn{11}{|l|}{ NMES } \\
\hline $\mathrm{T} 1$ & $\leq 80 \cdot 2$ & 1.00 & & 1.00 & & $\leq 14.8$ & 1.00 & & 1.00 & \\
\hline T2 & $80 \cdot 2-102 \cdot 6$ & 1.24 & $0.97,1.59$ & $1 \cdot 14$ & $0.84,1.55$ & $14.8-19.9$ & 1.58 & $1 \cdot 25,2 \cdot 01$ & 1.48 & $1.10,1.99$ \\
\hline T3 & $\geq 102 \cdot 6$ & $2 \cdot 20$ & $1 \cdot 68,2 \cdot 86$ & 1.65 & 1.18. $2 \cdot 30$ & $\geq 20 \cdot 0$ & 2.57 & $1.98,3.33$ & 1.84 & $1 \cdot 28,2 \cdot 64$ \\
\hline$P$ (trend) & & $<0.001$ & & 0.004 & & & $<0.001$ & & 0.001 & \\
\hline \multicolumn{11}{|c|}{ Intrinsic and milk sugars } \\
\hline $\mathrm{T} 1$ & $\leq 46.9$ & 1.00 & & 1.00 & & $\leq 9.3$ & 1.00 & & 1.00 & \\
\hline T2 & $47 \cdot 0-62 \cdot 8$ & 0.35 & $0.26,0.47$ & 0.52 & $0.36,0.75$ & $9 \cdot 3-12 \cdot 6$ & 0.37 & $0.28,0.49$ & 0.54 & $0.38,0.77$ \\
\hline T3 & $\geq 62 \cdot 8$ & 0.27 & $0.20,0.37$ & 0.42 & $0.29,0.61$ & $\geq 12 \cdot 6$ & 0.25 & $0.18,0.35$ & 0.41 & $0.28,0.60$ \\
\hline$P$ (trend) & & $<0.001$ & & $<0.001$ & & & $<0.001$ & & $<0.001$ & \\
\hline \multicolumn{11}{|l|}{ Sucrose } \\
\hline T1 & $\leq 64 \cdot 2$ & 1.00 & & 1.00 & & $\leq 11.9$ & 1.00 & & 1.00 & \\
\hline T2 & $64 \cdot 2-77 \cdot 6$ & 1.15 & $0.87,1.52$ & 1.03 & $0.72,1.45$ & $12 \cdot 0-15 \cdot 1$ & 1.40 & $1.05,1 \cdot 87$ & 1.26 & $0.83,1.90$ \\
\hline T3 & $\geq 77.6$ & 1.79 & $1 \cdot 38,2 \cdot 33$ & 1.42 & $0.99,2.03$ & $\geq 15 \cdot 1$ & 1.99 & $1.52,2.62$ & 1.54 & $1.08,2.19$ \\
\hline$P$ (trend) & & $<0.001$ & & 0.054 & & & $<0.001$ & & 0.018 & \\
\hline \multicolumn{11}{|l|}{ Fructose } \\
\hline $\mathrm{T} 1$ & $\leq 20 \cdot 0$ & 1.00 & & 1.00 & & $\leq 3.9$ & 1.00 & & 1.00 & \\
\hline T2 & $20 \cdot 0-27 \cdot 2$ & 0.71 & $0.53,0.94$ & 0.77 & $0.54,1 \cdot 10$ & $3.9-5 \cdot 4$ & 0.66 & $0.50,0.89$ & 0.74 & $0.54,1.02$ \\
\hline T3 & $\geq 27 \cdot 3$ & 0.69 & $0.51,0.94$ & 0.77 & $0.53,1.11$ & $\geq 5.4$ & 0.62 & $0.46,0.82$ & 0.69 & $0.49,0.97$ \\
\hline$P$ (trend) & & 0.019 & & $0 \cdot 161$ & & & 0.001 & & 0.036 & \\
\hline \multicolumn{11}{|l|}{ Glucose } \\
\hline T1 & $\leq 19 \cdot 6$ & 1.00 & & 1.00 & & $\leq 3.7$ & 1.00 & & 1.00 & \\
\hline T2 & $19 \cdot 6-25 \cdot 4$ & 0.76 & $0.57,1.02$ & 0.84 & $0.59,1.20$ & $3.7-5.0$ & 0.77 & $0.58,1.02$ & 0.78 & $0.55,1.09$ \\
\hline T3 & $\geq 25.4$ & 0.92 & $0.69,1.24$ & 1.00 & $0.70,1.42$ & $\geq 5 \cdot 0$ & 0.94 & $0.70,1.26$ & 0.97 & $0.69,1.37$ \\
\hline$P$ (trend) & & 0.577 & & 0.992 & & & 0.681 & & 0.850 & \\
\hline \multicolumn{11}{|l|}{ Lactose } \\
\hline $\mathrm{T} 1$ & $\leq 16 \cdot 1$ & 1.00 & & 1.00 & & $\leq 3.0$ & 1.00 & & 1.00 & \\
\hline T2 & $16 \cdot 1-23 \cdot 7$ & 0.50 & $0.39,0.64$ & 0.57 & $0.41,0.79$ & $3 \cdot 1-4 \cdot 7$ & 0.59 & $0.44,0.77$ & 0.65 & $0.48,0.89$ \\
\hline T3 & $\geq 23.7$ & 0.42 & $0.32,0.56$ & 0.63 & $0.45,0.86$ & $\geq 4.7$ & 0.45 & $0.34,0.60$ & 0.67 & $0.49,0.92$ \\
\hline$P$ (trend) & & $<0.001$ & & 0.004 & & & $<0.001$ & & 0.013 & \\
\hline \multicolumn{11}{|l|}{ Maltose } \\
\hline $\mathrm{T} 1$ & $\leq 3.5$ & 1.00 & & 1.00 & & $\leq 0.7$ & 1.00 & & 1.00 & \\
\hline T2 & $3.5-4.5$ & 0.95 & $0.73,1.23$ & 0.88 & $0.63,1.23$ & $0.7-0.9$ & 1.00 & $0.77,1.30$ & 0.89 & $0.64,1.25$ \\
\hline T3 & $\geq 4.5$ & 1.75 & $1 \cdot 30,2 \cdot 37$ & 1.22 & $0.83,1.79$ & $\geq 0.9$ & 1.79 & $1.31,2.44$ & 1.22 & $0.82,1.82$ \\
\hline$P$ (trend) & & $<0.001$ & & 0.292 & & & $<0.001$ & & 0.318 & \\
\hline
\end{tabular}

T, tertile; NMES, non-milk extrinsic sugars; SIMD, Scottish index of multiple deprivation.

*Energy-adjusted intake.

was used to define cut-offs for tertiles.

to 
Table 3. Food consumption and risk of having had treatment for decay $(n 1329)$ (Odds ratios and $95 \%$ confidence intervals)

\begin{tabular}{|c|c|c|c|c|c|}
\hline Tertile & Intake* $(g / d)$ & Crude OR & $95 \% \mathrm{Cl}$ & Adjusted OR $†$ & $95 \% \mathrm{Cl}$ \\
\hline \multicolumn{6}{|c|}{ Soft drinks (not diet) } \\
\hline $\mathrm{T} 1$ & $\leq 113 \cdot 1$ & 1.00 & & 1.00 & \\
\hline T2 & $113 \cdot 1-273 \cdot 1$ & $1 \cdot 16$ & $0.89,1.50$ & 1.08 & $0.76,1.55$ \\
\hline T3 & $\geq 273 \cdot 1$ & $2 \cdot 31$ & $1 \cdot 65,3 \cdot 23$ & 1.59 & $1 \cdot 08,2 \cdot 34$ \\
\hline$P$ (trend) & & $<0.001$ & & 0.022 & \\
\hline \multicolumn{6}{|c|}{ Confectionery } \\
\hline T1 & $\leq 18.5$ & 1.00 & & 1.00 & \\
\hline T2 & $18.5-34.5$ & 1.31 & $0.98,1.74$ & 1.38 & $0.96,1.99$ \\
\hline T3 & $\geq 34.5$ & $2 \cdot 67$ & $2.02,3.52$ & 1.97 & $1 \cdot 39,2 \cdot 78$ \\
\hline$P$ (trend) & & $<0.001$ & & $<0.001$ & \\
\hline \multicolumn{6}{|c|}{ Biscuits, cakes and pastries } \\
\hline $\mathrm{T} 1$ & $\leq 31.7$ & 1.00 & & 1.00 & \\
\hline T2 & $31 \cdot 7-51 \cdot 2$ & 0.93 & $0.72,1.21$ & 1.16 & $0.84,1.61$ \\
\hline T3 & $\geq 51 \cdot 3$ & 1.32 & $0.94,1.86$ & 1.45 & $0.99,2 \cdot 13$ \\
\hline$P$ (trend) & & 0.111 & & 0.054 & \\
\hline \multicolumn{6}{|c|}{ Yogurt and fromage frais } \\
\hline $\mathrm{T} 1$ & $\leq 51 \cdot 6$ & 1.00 & & 1.00 & \\
\hline $\mathrm{T} 2$ & $51 \cdot 6-123 \cdot 4$ & 0.51 & $0.38,0.69$ & 0.73 & $0.51,1.06$ \\
\hline T3 & $\geq 123 \cdot 4$ & 0.52 & $0.39,0.69$ & 0.99 & $0.69,1.43$ \\
\hline$P$ (trend) & & $<0.001$ & & 0.932 & \\
\hline \multicolumn{6}{|c|}{ Fruit (excluding fruit juice) } \\
\hline $\mathrm{T} 1$ & $\leq 102 \cdot 3$ & 1.00 & & 1.00 & \\
\hline T2 & $102 \cdot 3-193 \cdot 1$ & 0.55 & $0.41,0.74$ & 0.86 & $0.58,1 \cdot 25$ \\
\hline T3 & $\geq 193 \cdot 1$ & 0.44 & $0.33,0.58$ & 0.76 & $0.54,1.08$ \\
\hline$P$ (trend) & & $<0.001$ & & $0 \cdot 127$ & \\
\hline \multicolumn{6}{|c|}{ Fruit juice (including smoothies) } \\
\hline $\mathrm{T} 1$ & $\leq 30.4$ & 1.00 & & 1.00 & \\
\hline T2 & $30.4-95.6$ & 0.77 & $0.59,1.00$ & 0.76 & $0.52,1 \cdot 10$ \\
\hline T3 & $\geq 95.6$ & 0.53 & $0.40,0.69$ & 0.55 & $0.37,0.80$ \\
\hline$P$ (trend) & & $<0.001$ & & 0.002 & \\
\hline \multicolumn{6}{|c|}{ Milk and cream } \\
\hline T1 & $\leq 184 \cdot 6$ & 1.00 & & 1.00 & \\
\hline $\mathrm{T} 2$ & $184 \cdot 6-365 \cdot 6$ & 0.58 & $0.43,0.77$ & 0.63 & $0.44,0.90$ \\
\hline T3 & $\geq 365 \cdot 6$ & 0.48 & $0.37,0.62$ & 0.66 & $0.47,0.91$ \\
\hline$P$ (trend) & & $<0.001$ & & 0.011 & \\
\hline \multicolumn{6}{|c|}{ Crisps and savoury snacks } \\
\hline $\mathrm{T} 1$ & $\leq 16 \cdot 6$ & 1.00 & & 1.00 & \\
\hline T2 & $16 \cdot 6-21 \cdot 0$ & 1.32 & $0.98,1.77$ & 1.51 & $1 \cdot 06,2 \cdot 14$ \\
\hline T3 & $\geq 21 \cdot 0$ & 1.80 & $1 \cdot 30,2 \cdot 50$ & 1.79 & $1 \cdot 19,2 \cdot 69$ \\
\hline$P$ (trend) & & 0.001 & & 0.006 & \\
\hline
\end{tabular}

T, tertile; SIMD, Scottish index of multiple deprivation.

*Energy-adjusted intake; a second decimal place was used to define cut-offs for tertiles.

†Adjusted for age, SIMD quintile, whether milk or second teeth, whether had treatment to stop decay (e.g. painting and/or sealing), age first brushed teeth, and frequency of tooth brushing; subjects with missing values excluded.

There was no significant association between risk of having had treatment for decay and intakes of biscuits, cakes and pastries; yogurt and fromage frais; or whole fruit following adjustment.

Table 4 shows the risk of having had treatment for decay associated with combinations of NMES intake and reported frequency of tooth brushing. Compared with children who reported brushing their teeth frequently and had the lowest NMES intake ( $\leq 14.8 \%$ food energy), children who reported brushing their teeth once a day or less and had the highest NMES intake ( $\geq 20.0 \%$ food energy) were at a threefold risk of having received treatment for decay (adjusted OR 3.39; $95 \%$ CI 1.97, 5.82). Although frequent tooth brushing attenuated the risk associated with the highest NMES intakes, children who reported brushing their teeth frequently were still at increased risk of having had treatment for decay if they were in the highest tertile of NMES intake (adjusted OR $1.67 ; 95 \%$ CI $1.09,2 \cdot 55)$.

\section{Discussion}

Intakes of NMES and sucrose were positively associated with the risk of having received treatment for decay, as were intakes of non-diet soft drinks and confectionery, which were the largest contributors to NMES intake. The lack of association between total sugar intake and having received treatment for decay, but clear association with NMES intake, supports earlier reports indicating that NMES is the component of foods which contributes to dental decay ${ }^{(2)}$.

Our finding that there was no association between total sugar intake and having had treatment for decay is consistent with the finding from a previous UK study in 12-year-old 
Table 4. Tooth brushing frequency, non-milk extrinsic sugars intake and risk of having had treatment for decay $(n 1321)$ (Odds ratios and $95 \%$ confidence intervals)

\begin{tabular}{|c|c|c|c|c|c|c|c|}
\hline \multirow[b]{2}{*}{ Tooth brushing frequency } & \multirow[b]{2}{*}{ Intake (\% food energy) ${ }^{*}$} & \multicolumn{2}{|c|}{ Treated for decay $(n)$} & \multirow[b]{2}{*}{ Crude OR } & \multirow[b]{2}{*}{$95 \% \mathrm{Cl}$} & \multirow[b]{2}{*}{ Adjusted OR† } & \multirow[b]{2}{*}{$95 \% \mathrm{Cl}$} \\
\hline & & Yes & No & & & & \\
\hline \multirow[t]{3}{*}{ At least twice a day } & $\mathrm{T} 1(\leq 14 \cdot 8)$ & 135 & 204 & 1.00 & & 1.00 & \\
\hline & T2 (14.8-19.9) & 158 & 166 & 1.41 & $1.08,1.84$ & 1.36 & $0.97,1.92$ \\
\hline & T3 $(\geq 20.0)$ & 166 & 117 & $2 \cdot 16$ & $1.54,3.04$ & 1.67 & $1.09,2.55$ \\
\hline \multirow[t]{3}{*}{ Once a day or less } & $\mathrm{T} 1(\leq 14.8)$ & 50 & 48 & 1.57 & $1.00,2.46$ & 1.34 & $0.74,2.43$ \\
\hline & T2 (14.8-19.9) & 82 & 43 & $3 \cdot 26$ & $2 \cdot 13,5.00$ & 2.64 & $1.58,4.41$ \\
\hline & T3 $(\geq 20 \cdot 0)$ & 114 & 38 & 4.99 & $3.15,7.88$ & $3 \cdot 39$ & $1.97,5.82$ \\
\hline
\end{tabular}

T, tertile; SIMD, Scottish index of multiple deprivation.

${ }^{*}$ A second decimal place was used to define cut-offs for tertiles.

†Adjusted for age, SIMD quintile, whether milk or second teeth, whether had treatment to stop decay (e.g. painting and/or sealing) and age first brushed teeth; subjects with missing values excluded.

children $^{(21)}$, but contrasts with an older UK study of children carried out in 1979-81, which found a positive (but low) correlation between total sugar intake and dental caries ${ }^{(22)}$. A recent review on sucrose and dental caries in children and adults aged $<35$ years concluded that there is no reliable relationship between the amount of sugar consumed and dental caries: only six of fifteen studies included in the review found a significant relationship between total sugar intake and dental caries ${ }^{(23)}$. Another systematic review of sugar consumption and dental caries in countries where there is 'moderate-to-extensive' fluoride exposure found that of thirty-six papers that scored $>55$ out of 100 on the evidence tables, only two papers found a 'strong relationship' between sugar intake and caries development, sixteen papers found a 'moderate relationship' and eighteen papers found a 'weak-to-no relationship'(24).

The positive association between crisps and savoury snacks and having had treatment for dental decay is supported by an English study, which found a strong correlation between crisp consumption and the number of decayed, missing or filled teeth $^{(25)}$. Although crisps and savoury snacks contribute less than $1 \%$ to NMES intake ${ }^{(13)}$, sticky/chewy foods leave residue on teeth that prolongs exposure to carbohydrate and results in a lower oral $\mathrm{pH}^{(26)}$. The severity of processing undergone by these snack foods and the type of flavouring agents with which they are coated may also influence the cariogenicity of these snacks, with certain varieties of crisps being almost as cariogenic as semi-sweet biscuits ${ }^{(27)}$. In addition, children who consume crisps and savoury snacks may be likely to consume them with non-diet soft drinks (Spearman's $r$ 0.19, $P<0.001)$ that are rich in sugar.

There were significant inverse associations between intakes of intrinsic and milk sugars, lactose, milk and cream and having had treatment for dental decay. Care should be taken when interpreting these associations as they may be partly due to the inverse correlation between NMES and intrinsic and milk sugars (Spearman's $r-0 \cdot 36, P<0 \cdot 001$ ) and lactose (Spearman's $r-0.21, P<0.001$ ), i.e. individuals with the highest NMES intakes are likely to have the lowest intakes of intrinsic and milk sugars. However, the inverse association with intrinsic and milk sugars remained after adjusting for NMES intake (data not shown). Milk and milk products are alkaline and contain calcium, phosphates and protein which may aid remineralisation of dental enamel following exposure to reduced $\mathrm{pH}^{(26)}$. Nevertheless, most yogurts and fromage frais should not be considered as protective against dental decay due to their added sugar content, which accounted for $10 \%$ of total NMES intake ${ }^{(13)}$.

There was no significant association between whole fruit intake and treatment for decay after adjusting for potential confounding factors, which supports the recommendation that foods rich in NMES be replaced with fresh fruit and vegetables $^{(2)}$. The inverse association with fruit juice (including smoothies) is surprising given that fruit juice contributes about $10 \%$ to NMES intake ${ }^{(13)}$; therefore, this finding should be interpreted with caution. There was no significant difference in NMES intake between the lowest and highest tertiles of fruit juice intake; therefore, it is likely that fruit juice intake is correlated with other dietary factors or behaviours that influence risk of dental caries. For example, fruit juice consumption was significantly inversely correlated with consumption of non-diet soft drinks (Spearman's $r-0 \cdot 10$, $P<0.001$ ) and crisps and savoury snacks (Spearman's $r-0 \cdot 20, P<0 \cdot 001)$, and positively correlated with milk and cream (Spearman's $r 0 \cdot 07, P=0 \cdot 016$ ) and yogurt and fromage frais (Spearman $r 0 \cdot 12, P<0 \cdot 001$ ).

Although frequent tooth brushing attenuated the risk associated with the highest NMES intakes, children who reported brushing their teeth frequently were still at increased risk of having received treatment for decay if they had the highest NMES intakes. Similarly, the National Diet and Nutrition Survey of children aged $1.5-4.5$ years carried out during 1992-3 found that the benefits of tooth brushing did not outweigh the detrimental effect of frequent sugar consumption $^{(28)}$. The benefits of tooth brushing are usually attributed to the use of fluoride toothpastes rather than the act of brushing ${ }^{(29)}$. The majority $(97 \%)$ of children in this survey used a fluoride toothpaste, and it is likely that the protective effect associated with frequent tooth brushing is due to the fluoride rather than to brushing alone.

The benefits of fluoride result mainly from its topical effect, i.e. direct contact with the teeth ${ }^{(30)}$. Fluoride present in saliva or in contact with teeth from toothpaste inhibits bacterial enzymes that produce acid in plaques, and aids remineralisation of the enamel ${ }^{(31)}$. Total fluoride intake depends on the fluoride concentration in the water supplies; however, within Scotland, there are no fluoridated areas except for a small pocket of naturally fluoridated water in rural Morayshire ${ }^{(32)}$. Tea leaves, 
and therefore tea infusions, are also a major dietary source of fluoride depending on the strength of the infusion.

A number of factors should be borne in mind when interpreting these results and when designing future studies of dental caries. In particular, (i) the frequency of consumption, (ii) the consumption of NMES or NMES-rich foods just before bedtime and (iii) the placement of NMES-rich foods within a meal may have influenced our observed associations, and this information was not captured by the FFQ. The frequency of eating confectionery and sugary foods was positively associated with caries in 12-year-old English schoolchildren $^{(21)}$, and in a recent review, frequency of sugar consumption was significantly associated with dental caries in nineteen of thirty-one studies ${ }^{(23)}$. The consumption of sugary foods and drinks just before bedtime has also been associated with caries prevalence ${ }^{(28,33,34)}$. During sleep, the salivary flow rate is negligible, and the protective effects of saliva (e.g. its buffering effect and antibacterial action) are lost ${ }^{(1,35,36)}$. Therefore, ideally the hour before bedtime should be a sugar-free time ${ }^{(33)}$, and the most important time to brush teeth is just before bedtime ${ }^{(35)}$. In addition, the position of the NMES-rich food within a meal can influence the fall in plaque $\mathrm{pH}^{(37)}$ and may therefore influence caries risk. The greatest benefit is thought to occur when 'sugary' and 'non-sugary' foods are consumed together, or when the sugary food is sandwiched between non-sugary foods ${ }^{(37)}$.

The present study is based on self-reported treatment for decay, and the results were not validated by access to dental case notes. Nevertheless, evidence exists to support the value of dental self-reported measures in epidemiological studies, with a high level of concordance found between child and parent/guardian reporting, and positive correlations found between children's self-report and clinically measured oral health ${ }^{(38)}$. However, societal pressure may influence parental reporting of frequency of tooth brushing, with a larger percentage of parents reporting that their child 'brushes twice or more per day' than the children ${ }^{(38)}$.

Information was not collected on the level of decay, such as the number of fillings or the number of teeth removed due to decay. Therefore, we could not differentiate between children with one filling and children with many fillings or many teeth removed due to decay. Additionally, it should be noted that untreated caries may be present in some of the children, and therefore the present study could only assess the risk of having received treatment for decay rather than the risk of decay itself. Information was also not collected on whether children were left to brush their teeth themselves, i.e. without parental/guardian supervision, which has been associated with caries prevalence ${ }^{(28)}$.

The cross-sectional nature of this survey makes it difficult to draw conclusions regarding cause and effect, and the harmful effects of NMES may even be underestimated. For example, current dental status may reflect earlier dietary patterns, and children with untreated dental caries may limit their sugar intake due to pain. Ideally, prospective studies are required to assess the influence of diet and lifestyle on the development of caries.

Although the association between NMES intake and dental health was highlighted in the 1989 report 'Dietary sugars and human disease ${ }^{(2)}$, and the recommendation that the population average intake should not exceed $10 \%$ of total energy was published in $1991^{(12)}$, the little evidence available on trends in NMES consumption in UK children suggests that NMES intake has not changed much in the last 20 years, and may even be increasing. Mean NMES intakes in 11-12year-old Northumbrian children were estimated to be $15.0 \%$ energy in $1980,16.9 \%$ energy in 1990 and $16.1 \%$ energy in $2000^{(39)}$. The National Diet and Nutrition Survey of young people aged 4-18 years estimated mean NMES intakes to be $16.7 \%$ of food energy in boys and $16.4 \%$ of food energy in girls in $1997^{(40)}$, which are slightly lower than the estimated intake of $17.4 \%$ of food energy in both boys and girls aged $3-17$ years in Scotland in $2006^{(13)}$

High intakes of NMES may also have implications for diseases other than dental caries, particularly for the present obesity epidemic, by contributing to the excess energy consumption responsible for obesity development. For example, greater consumption of sugar-sweetened beverages, which are the largest contributors to NMES intake (23\% in the present study), has been associated with weight gain and obesity in both adults and children ${ }^{(41)}$.

The recommended intake for NMES of less than $10 \%$ of total energy ( $11 \%$ of food energy) in children was originally intended to be achieved in 2005 , though the time scale was later extended to 2010. It is unlikely that the NMES target will be met, given that the mean intake in 2006 was $17.4 \%$ food energy ${ }^{(13)}$. Although removing all non-diet soft drinks from the diet and replacing the energy by increasing the intake of fruit and vegetables will lead to some reduction in NMES intake (based on the mean intakes of the group), consumption of confectionery, biscuits, cakes, pastries, yogurt and fromage frais would have to decrease and intake of starchy foods would have to increase before the recommendation for NMES intake is met in children in Scotland ${ }^{(42)}$.

Within Scotland, an action plan was introduced in 2005 for improving dental health with targets set for 5 year olds and $11 / 12$ year olds in $2010^{(43)}$. The action plan had a particular focus on improving the oral health of children, including extension of tooth brushing schemes to primary schools with the poorest oral health records and promotion of oral health in secondary schools. Targets for older children are lacking, and prevention of caries in children aged under 5 years should also be a priority as a recent report published by the Audit Commission ${ }^{(44)}$ highlighted a widening gap in the mean number of decayed, missing and filled teeth per 5-year-old child between those living in deprived communities and those living in the rest of England.

The highest grades of recommendations for primary prevention of caries are for (1) dental health education advice, (2) brushing teeth twice a day using a toothpaste with at least $1000 \mathrm{ppm}$ fluoride and (3) application and maintenance of sealants in tooth pits or fissures (grade A) rather than changing dietary intake (grades B and C) ${ }^{(1)}$. However, the present study suggests that increasing the frequency of tooth brushing, while reducing the risk of developing caries, will not compensate for a high intake of NMES. Therefore, to improve dental health in children, dental health strategies must continue to stress the importance of both good oral hygiene and reduced NMES intake.

\section{Acknowledgements}

G. McN., L. F. M. and C. S. conceived the original study with input from L. C. A. C., J. I. M. and B. A. H. L. F. M. 
conducted the statistical analysis, with contributions from A. B., and drafted the manuscript, which was reviewed by all the authors. The authors state that there are no conflicts of interest. The study was funded by the Food Standards Agency, Scotland (contract S14029).

\section{References}

1. Scottish Intercollegiate Guidelines Network (2000) Preventing Dental Caries in Children at High Caries Risk: Targeted Prevention of Dental Caries in the Permanent Teeth of 6-16 Year Olds Presenting for Dental Care. SIGN Publication no. 47. Edinburgh: Scottish Intercollegiate Guidelines Network.

2. Department of Health (1989) Dietary Sugars And Human Disease. Report on Health And Social Subjects: 37. London: HMSO.

3. Scottish Executive (2005) Towards Better Oral Health in Children: An Independent Analysis Of Responses to the Consultation on Children's Oral Health. Edinburgh: Scottish Executive.

4. Gibson S \& Williams S (1999) Dental caries in pre-school children: associations with social class, toothbrushing habit and consumption of sugars and sugar-containing foods. Caries Res 33, 101-113.

5. Levin KA \& Currie C (2009) Inequalities in toothbrushing among adolescents in Scotland 1998-2006. Health Educ Res 24, 87-97.

6. Maes L, Vereecken C, Vanobbergen J, et al. (2006) Tooth brushing and social characteristics of families in 32 countries. Int Dent J 56, 156-167.

7. Pitts NB, Boyles J, Nugent ZJ, et al. (2006) The dental caries experience of 11-year-old children in Great Britain. Surveys coordinated by the British Association for the Study of Community Dentistry in 2004/2005. Community Dent Health 23, $44-57$.

8. Pitts NB, Boyles J, Nugent ZJ, et al. (2007) The dental caries experience of 5-year-old children in Great Britain (2005/6). Surveys coordinated by the British Association for the Study of Community Dentistry. Community Dent Health 24, 59-63.

9. Bromley C, Sproston K \& Shelton N (editors) (2005) The Scottish Health Survey 2003. Edinburgh: The Stationery Office.

10. ISD Scotland (2009) Dental: fees. http://www.isdscotland.org/ isd/4682.html (accessed July 2009).

11. The Scottish Office Department of Health (1996) Eating for Health: A Diet Action Plan for Scotland. Edinburgh: The Scottish Office.

12. Department of Health (1991) Dietary Reference Values for Food Energy and Nutrients for the United Kingdom. Report of the Panel on Dietary Reference Values of the Committee on Medical Aspects of Food Policy. Report on Health and Social Subjects no. 41. London: HMSO.

13. Sheehy C, McNeill G, Masson L, et al. (2008) Survey of Sugar Intake Among Children in Scotland Aberdeen: Food Standards Agency Scotland. http://www.food.gov.uk/multimedia/ pdfs/sugarintakescot2008rep.pdf

14. Scottish Collaborative Group (2009) Food frequency questionnaire. http://www.foodfrequency.org.uk (accessed September 2009).

15. Nelson M, Erens B, Bates B et al. (editors) (2007) Low Income Diet and Nutrition Survey. London: The Stationery Office. http:// www.food.gov.uk/science/dietarysurveys/lidnsbranch/

16. National Diet and Nutrition Survey (2004) Adults aged 19 to 64 years: Technical Report: Appendix H. London: The Stationery Office.

17. Scottish Index of Multiple Deprivation (2006) Technical Report. Edinburgh, Office of the Chief Statistician: Scottish Executive. http://www.scotland.gov.uk/Publications/2006/10/13142913/0
18. Cole TJ, Freeman JV \& Preece MA (1995) Body mass index reference curves for the UK, 1990. Arch Dis Child 73, 25-29.

19. Rao JNK \& Scott AJ (1984) On chi-squared tests for multiway contingency tables with proportions estimated from survey data. Ann Stat 12, 46-60.

20. Willett W (1998) Nutritional Epidemiology, 2nd ed. New York: Oxford University Press.

21. Beighton D, Adamson A \& Rugg-Gunn A (1996) Associations between dietary intake, dental caries experience and salivary bacterial levels in 12-year-old English schoolchildren. Archs Oral Biol 41, 271-280.

22. Rugg-Gunn AJ, Hackett AF, Appleton DR, et al. (1984) Relationship between dietary habits and caries increment assessed over two years in 405 English adolescent school children. Arch Oral Biol 12, 983-992.

23. Anderson CA, Curzon MEJ, van Loveren C, et al. (2009) Sucrose and dental caries: a review of the evidence. Obes Rev 10, Suppl. 1, 41-54.

24. Burt BA \& Satishchandra P (2001) Sugar consumption and caries risk: a systematic review. J Dent Educ 65, 1017-1023.

25. Maliderou M, Reeves S \& Noble C (2006) The effect of social demographic factors, snack consumption and vending machine use on oral health of children living in London. $\mathrm{Br}$ Dent $J$ 201, 441-444.

26. Webster-Gandy J, Madden A \& Holdsworth M (editors) (2006) Oxford Handbook of Nutrition and Dietetics. Oxford: Oxford University Press.

27. Grenby TH (1990) Snack foods and dental caries. Investigations using laboratory animals. Br Dent $J \mathbf{1 6 8}, 353-361$.

28. Moynihan PJ \& Holt RD (1996) The national diet and nutrition survey of 1.5 to 4.5 year old children: summary of the findings of the dental survey. Br Dent $J \mathbf{1 8 1}, 328-332$.

29. Sutcliffe P (1983) Oral cleanliness and dental caries. In The Prevention of Denal Caries, pp. 159-174 [JJ Murray, editor]. Oxford: Oxford University Press.

30. Centers for Disease Control and Prevention (2001) Recommendations for using fluoride to prevent and control dental caries in the United States. MMWR Recomm Rep 50, 1-42.

31. Truswell S (2007) Fluoride. In Essentials of Human Nutrition, 3rd ed., pp. 159-162 [J Mann and S Truswell, editors]. New York: Oxford University Press.

32. Jones S \& Lennon K (2005) One in a Million: Facts About Water Fluoridation, 2nd ed. London: The British Fluoridation Society. http://www.bfsweb.org/onemillion/onemillion.htm

33. Levine RS (2001) Caries experience and bedtime consumption of sugar-sweetened foods and drinks - a survey of 600 children. Community Dent Health 18, 228-231.

34. Levine RS, Nugent ZJ, Rudolf MCJ, et al. (2007) Dietary patterns, toothbrushing habits and caries experience of schoolchildren in West Yorkshire, England. Community Dent Health 24, 82-87.

35. Dawes C (2008) Salivary flow patterns and the health of hard and soft oral tissues. J Am Dent Assoc 139, 18S-24S.

36. Schneyer LH, Pigman W, Hanahan L, et al. (1956) Rate of flow of human parotid, sublingual and submaxillary secretions during sleep. J Dent Res 35, 109-114.

37. Rugg-Gunn AJ, Edgar WM \& Jenkins GN (1981) The effect of altering the position of a sugary food in a meal upon plaque $\mathrm{pH}$ in human subjects. J Dent Res 60, 867-872.

38. Jamieson LM, Thomson WR \& McGee R (2004) An assessment of the validity and reliability of dental self-report items used in a National Child Nutrition Survey. Community Dent Oral Epidemiol 32, 49-54.

39. Rugg-Gunn AJ, Fletcher ES, Matthews JNS, et al. (2007) Changes in consumption of sugars by English adolescents over 20 years. Public Health Nutr 10, 354-363. 
40. Gregory J, Lowe S, Bates CJ, et al. (2000) National Diet and Nutrition Survey: Young People Aged 4 to 18 Years. London: The Stationery Office.

41. Malik VS, Schulze MB \& Hu FB (2006) Intake of sugarsweetened beverages and weight gain: a systematic review. Am J Clin Nutr 84, 274-288.

42. McNeill G, Masson LF, Craig LCA, et al. (2009) Sugar and fat intake among children in Scotland: what is needed to reach the dietary targets? Public Health Nutr (Epublication ahead of print version 8 December 2009).

43. Scottish Executive (2005) An Action Plan for Improving Oral Health and Modernising NHS Dental Services in Scotland. Edinburgh: Scottish Executive.

44. Audit Commission (2010) Giving Children a Health Start. Health Report, February 2010. London: Audit Commission. 